\title{
Microscopic Model of Heteroepitaxy of GaAs on Sil(100)
}

\section{Citation}

Kaxiras, Efthimios, Oscar L. Alerhand, John D. Joannopoulos, and George W. Turner. 1989. Microscopic model of heteroepitaxy of GaAs on Si(100). Physical Review Letters 62, no. 21: 2484-2486.

\section{Published Version}

http://dx.doi.org/10.1103/PhysRevLett.62.2484

\section{Permanent link}

http://nrs.harvard.edu/urn-3:HUL.InstRepos:10605460

\section{Terms of Use}

This article was downloaded from Harvard University's DASH repository, and is made available under the terms and conditions applicable to Other Posted Material, as set forth at http:// nrs.harvard.edu/urn-3:HUL.InstRepos:dash.current.terms-of-use\#LAA

\section{Share Your Story}

The Harvard community has made this article openly available.

Please share how this access benefits you. Submit a story.

Accessibility 


\title{
Microscopic Model of Heteroepitaxy of GaAs on Si(100)
}

\author{
Efthimios Kaxiras, ${ }^{(1)}$ O. L. Alerhand, ${ }^{(2)}$ J. D. Joannopoulos, ${ }^{(2)}$ and G. W. Turner ${ }^{(3)}$ \\ (1) IBM Research Division, T. J. Watson Research Center, Yorktown Heights, New York 10598 \\ ${ }^{(2)}$ Department of Physics, Massachusetts Institute of Technology, Cambridge, Massachusetts 02139 \\ ${ }^{(3)}$ Lincoln Laboratory, Massachusetts Institute of Technology, Lexington, Massachusetts 02173
}

(Received 21 November 1988)

\begin{abstract}
A new microscopic model of heteroepitaxial growth is introduced using $\mathrm{GaAs}$ on $\mathrm{Si}(100)$ as a prototype. This model takes into account specific features of surface topology, predicts that in the prototype system conventional two-dimensional epitaxy should be inhibited, and provides a fundamental explanation for the three-dimensional nature of the initial stages of growth. The ingredients of the model, which are supported by total-energy calculations, include new structural geometries for each stage of growth and the chemical and rehybridization reactions linking these stages.
\end{abstract}

PACS numbers: $68.55 .-\mathrm{a}, 81.10 .-\mathrm{h}$

Epitaxial growth of dissimilar semiconductor materials holds significant potential for technological applications and has been the subject of major international efforts in recent years. ${ }^{1}$ Nevertheless, relatively little theoretical work has been performed to understand the fundamental interactions governing the initial stages of growth and the structure of the first few monolayers in these systems. Of particular interest is the prototypical system involving growth of $\mathrm{GaAs}$ on $\mathrm{Si}(100)$ substrates. Experimental studies have shown that on slightly miscut (vicinal) Si surfaces GaAs initially grows in an islandlike or three-dimensional mode in the vicinity of stationary steps. ${ }^{2-4}$ This is in contrast to both the usual planar or two-dimensional epitaxial mode which proceeds by step motion, as well as the conventional three-dimensional modes driven by strain or absence of wetting. ${ }^{5}$ In this paper we present a microscopic theoretical model which, for the first time, can explain the fundamental mechanism for this new type of three-dimensional heteroepitaxial growth. The model, supported by total-energy calculations within a tight-binding formalism, provides a stage-by-stage description of growth on surface steps, including the driving chemical and rehybridization reactions. It clearly shows the crucial role of double-layer steps (DLS) on the Si surface in initiating the growth of three-dimensional GaAs islands and inhibiting layered epitaxial growth. The only previous attempt at a microscopic description of growth has concentrated on the flat regions of the Si substrate. ${ }^{6}$ To our knowledge, DLS on the $\mathrm{Si}$ surface have so far been considered only in the context of eliminating antiphase domains in GaAs overlayers (for a review see Ref. 6).

The essential physical and chemical concepts which underlie the construction of our model are based on the following observations. First, both $\mathrm{Ga}$ and As can form passivated structures with threefold or fourfold coordination. When $\mathrm{Ga}$ and As atoms are fourfold coordinated, it is important that they form nearest-neighbor pairs to satisfy charge neutrality requirements. Threefold coordinated $\mathrm{Si}$ on the other hand typically has an energeti- cally unfavorable dangling bond. Second, a recent $a b$ initio theoretical investigation ${ }^{7}$ has shown that on flat regions of the $\mathrm{Si}(100)$ surface a mixed $\mathrm{GaAs}$ overlayer phase (formed by switching like-atom bonds to unlikeatom bonds) is energetically favored and inhibits further growth of bulk GaAs along the [100] direction. Thus, there is a strong tendency for mixing at the initial stages which needs to be overcome in any viable model of growth. Finally, idealized growth of GaAs along the [100] direction would lead to physically unacceptable large electric fields due to a net interface charge. ${ }^{8}$

With these ideas in mind, we proceed now to introduce the model of growth. The discussion will follow a realistic sequence of the growth stages as As and Ga atoms are deposited on the Si surface. We begin with the clean $\mathrm{Si}$ surface, which when cut slightly off axis from the (100) orientation, necessarily contains steps. Experimentally it is observed that vicinal $\mathrm{Si}(100)$ surfaces under usual MBE growth conditions contain steps with height ranging from one to several atomic layers. ${ }^{2,9}$ Of the various possible step configurations, we focus on the DLS, which have the appealing feature that they naturally eliminate antiphase domains. Moreover, theoretically ${ }^{10}$ it is found that DLS are the lowest-energy steps, and indeed surfaces can be prepared with only these steps present. ${ }^{11}$ Theoretical investigations ${ }^{10,12}$ indicate that the lowest-energy configuration for a DLS is as shown in Fig. 1(a). On this step there is a row of threefold-coordinated $\mathrm{Si}$ atoms (shown with darker shading) which do not participate in dimer formation. As such, they are more reactive and less stable energetically.

In the usual experimental setup for MBE growth, the ambient pressure of As leads to an As-covered Si surface even before atomic beams are supplied for growth. On the flat regions of the Si substrate the As atoms break the existing Si dimers and form new As dimers. ${ }^{13}$ The ensuing threefold coordination of the As atoms and fourfold coordination of the underlying $\mathrm{Si}$ atoms is electronically passive and energetically very stable. To obtain the optimal configuration, however, one has to allow for 
(a)

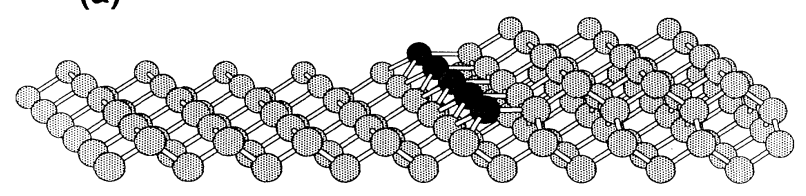

(b)

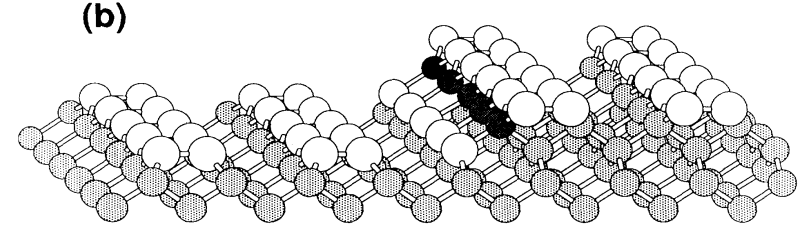

(c)

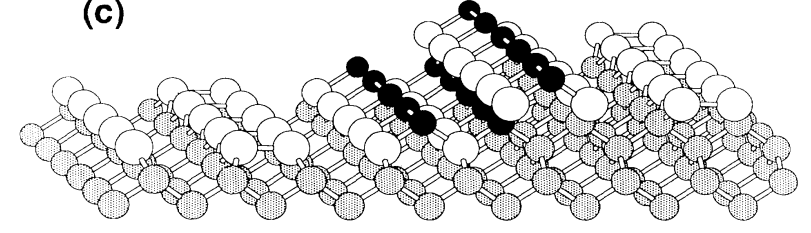

FIG. 1. The stages of growth. (a) The clean $\mathrm{Si}(100)$ surface with a double-layer step. The Si dimers on either side of the step are oriented parallel to the step. The threefoldcoordinated $\mathrm{Si}$ atoms at the step shown by a darker shading do not participate in dimer formation and should be more reactive. (b) The As-covered configuration. The row of threefoldcoordinated $\mathrm{Si}$ atoms at the step of the clean surface have been substituted by As atoms. The displaced Si atoms (shown by darker shading) are now fourfold coordinated and are bonded to three surface As atoms. (c) The Ga- and As-covered step configuration. Note that adding more $\mathrm{Ga}$ atoms on either side of the step would permit mixing which would inhibit (100) growth. Thus preferred growth proceeds by growing larger and larger overlayers directly on the step along the [211] direction.

proper bonding of the row of threefold-coordinated $\mathrm{Si}$ atoms at the steps. We propose that this can be achieved by interchanging these threefold-coordinated $\mathrm{Si}$ atoms with As atoms, and incorporating the appropriate amounts of As to complete the coverage of the surface. This substitution leads to the novel configuration shown in Fig. 1(b). This geometry is very appealing for the following reasons. First, in the final configuration all $\mathrm{Si}$ atoms are fourfold coordinated and all As atoms are threefold coordinated, which results in a passive and stable structure. Second, prior to the substitution, the $\mathrm{Si}$ atoms at the step are exposed and threefold coordinated, much like in the ideal $\mathrm{Si}(111)$ surface. In the latter case it has been shown that it is favorable for As atoms to replace the entire uppermost layer of $\mathrm{Si}$ atoms. ${ }^{14}$ [In a different context substitution of $\mathrm{As}$ for $\mathrm{Si}$ atoms has also been proposed ${ }^{6}$ to reduce polarity effects in layered growth on flat regions of $\mathrm{Si}(100)$.] Thus we believe the configuration shown in Fig. 1(b) is most likely the equi- librium ground state of the As-covered vicinal $\mathrm{Si}(100)$ surface, and should be experimentally observable.

The next step in the growth model consists of depositing $\mathrm{Ga}$ on the surface. In the flat regions of the surface, the $\mathrm{Ga}$ atoms will break the As-As bonds and form a very low-energy mixed bilayer, with equal amounts of $\mathrm{Ga}$ and As atoms in each atomic layer. ${ }^{7}$ This mixed bilayer has a structure resembling wurtzite under large inhomogeneous strain. Reverting from this phase to the stable zinc-blende structure of GaAs [alternating (100) planes composed exclusively of $\mathrm{Ga}$ or As] is inhibited by a large energy barrier. Thus, growth of zinc-blende $\mathrm{GaAs}$ on the flat regions of $\mathrm{Si}(100)$ is suppressed. ${ }^{7}$

The new element in the present approach is the influence of the step topology which prevents mixing in the immediate neighborhood of the steps and eventually promotes three-dimensional growth. To see this, first consider the row of $\mathrm{Si}$ atoms at the step in which each $\mathrm{Si}$ atom is surrounded by three As atoms as shown in Fig. 1(b). Electronegativity arguments ${ }^{15}$ indicate that it is energetically favorable to replace these $\mathrm{Si}$ atoms by $\mathrm{Ga}$ atoms. The removed $\mathrm{Si}$ atoms are allowed to diffuse to bulk positions of lower energy, or to equivalent surface sites. The Ga-for-Si substitution creates pairs of fourfold-coordinated $\mathrm{Ga}$ and $\mathrm{As}$ atoms at the step edge, as shown in Fig. 1(c). Additional Ga atoms are incorporated between As-As bonds on either side of the step. Each $\mathrm{Ga}$ atom becomes threefold coordinated, bonding to two As and one Ga. The As atoms on either side of the step remain threefold coordinated. Thus, the new structure is again electronically passive. But now, mixing is not energetically favorable to occur in this or any other subsequent overlayer configuration. This is because mixing at the step configuration cannot create more $\mathrm{Ga}-\mathrm{As}$ bonds, in place of $\mathrm{Ga}-\mathrm{Ga}$ or As-As bonds, which is the driving mechanism for mixing at the terraces. ${ }^{7}$ Avoidance of mixing is crucial if the growth of bulklike GaAs is to continue. The structural feature which leads to elimination of mixing is the creation of the Ga-As fourfold-coordinated pairs at the edge of the step. This feature is a direct consequence of the geometry of the As-covered step configuration described earlier in Fig. 1(b). Therefore, the proposed As-covered configuration is not only intrinsically very stable, but crucial in promoting growth on the step. We emphasize that this is very different from the typical epitaxial growth process where adsorbed atoms are continuously incorporated at highly reactive propagating steps.

To substantiate our claims about the stability of the $\mathrm{Ga}$ and As covered step configuration, we have performed total-energy calculations in a tight-binding formalism, which permit proper treatment of rehybridization effects upon atomic relaxation. ${ }^{16}$ In this method the total energy is the sum of the band structure and screened ionic repulsion energies. The latter are obtained by fitting to a variety of configurations, for which the total energy is known from $a b$ initio calculations. ${ }^{7}$ 
Band-structure parameters are taken from Harrison's solid-state table of elements. ${ }^{17}$ This formalism is used to compare the energy of the relaxed step configuration shown in Fig. 1(c) with the optimal arrangement of the same number of $\mathrm{Ga}$ and As atoms on a flat region (terrace) of the $\mathrm{Si}$ surface. The terrace configuration involves locally mixed $\mathrm{Ga}$ and As layers. ${ }^{7}$ Since the ratio of $\mathrm{Ga}$ to As atoms at the step is not 1:1, the corresponding terrace configuration cannot be fully mixed. Nevertheless, we have included the full energy per GaAs pair gained upon mixing, ${ }^{7}$ thereby deliberately underestimating the energy of the terrace configuration. The final result is that the energy of the step configuration is lower than the energy of the terrace configuration by $\sim 1.5 \mathrm{eV}$ per unit length $(a \sqrt{2})$ along the step. A measure of the reliability of this estimate comes from the fact that the energy difference does not change when the step and terrace configurations are extended to include one more row of $\mathrm{Ga}$ dimers.

Let us now conclude by describing some further consequences of the growth model being proposed. We note that the exposed plane at the step is no longer the (100) plane of GaAs, but rather the (211) plane. This plane is nonpolar, and has been proposed as an alternate orientation for the Si substrate ${ }^{18}$ in order to eliminate interface charges associated with GaAs growth along the [100] direction. It is then reasonable to assume that growth will proceed along a direction perpendicular to the exposed plane at the step, rather than along [100]. This leads to three-dimensional growth in a direction oblique to the surface, and the formation of GaAs islands nucleated at surface DLS, in agreement with experimental indications. $^{2-4}$ When these islands coalesce, thick layers of $\mathrm{GaAs}$ are obtained. Residual polarity problems at the base of the islands can be relieved by local intermixing as has been suggested before. ${ }^{8}$ We emphasize that no special cut is needed to promote this growth, but only a sufficient density of DLS on the surface which is readily obtained by slight misorientation from the (100) axis. Nevertheless, a special cut corresponding to the largest density of DLS is the (311) plane. Of course, determination of the optimal cut is rather difficult, requiring the consideration of additional issues including strains, defects, and temperature. Thus, a different misorientation such as (511) or (711) may prove to be optimal.

In summary, we have proposed a new model for GaAs growth on vicinal $\mathrm{Si}(100)$ surfaces. The model is based on DLS on the Si surface acting as nucleation sites for GaAs islands. A stage-by-stage justification of the stepnucleated growth is presented, including some of the details of the associated reactions. An As-covered step configuration is identified, which is exceptionally stable, and is crucial in eliminating mixing at the neighborhood of the step. Total-energy calculations within a tightbinding formalism show that the unmixed step configuration is energetically favored over the optimal mixed terrace configuration. It is concluded from the above arguments that three-dimensional island growth is promoted along the [211] direction perpendicular to the step planes. This also has the appealing property of eliminating the formation of dipolar fields. The coalescence of the islands leads to thick, bulklike, GaAs layers. The usual islands of dislocations and lattice strain associated with these thick layers lie beyond the scope of our work and have not been addressed here.

This work was supported in part by Joint Services Electronics Program, Contract No. DAAL 03-86-K0002 .

${ }^{1}$ See, e.g., Heteroepitaxy in Silicon, edited by J. C. C. Fan and J. M. Poate, MRS Symposia Proceedings No. 67 (Materials Research Society, Pittsburgh, PA, 1986); Heteroepitaxy in Silicon II, edited by J. C. C. Fan, J. M. Phillips, and B.-Y. Tsaur, MRS Symposia Proceedings No. 91 (Materials Research Society, Pittsburgh, PA, 1987).

${ }^{2}$ S. Nishi, H. Inomata, M. Akiyama, and K. Kaminishi, Jpn. J. Appl. Phys. 24, L391 (1985).

${ }^{3}$ R. Hull and A. Fischer-Colbrie, Appl. Phys. Lett. 50, 851 (1987).

${ }^{4}$ D. K. Biegelsen, F. A. Ponce, A. J. Smith, and J. C. Tramontana, J. Appl. Phys. 61, 1856 (1987).

${ }^{5}$ J. A. Venables, G. D. T. Spiller, and M. Hanbucken, Rep. Prog. Phys. 47, 399 (1984).

${ }^{6}$ H. Kroemer, J. Cryst. Growth 81, 193 (1987).

${ }^{7}$ E. Kaxiras and J. D. Joannopoulos (to be published). The energy gained by mixing is $1.3 \mathrm{eV}$ per GaAs dimer.

${ }^{8}$ W. A. Harrison, E. A. Kraut, J. R. Waldrop, and R. W. Grant, Phys. Rev. B 18, 4402 (1978).

${ }^{9}$ P. R. Pukite and P. I. Cohen, Appl. Phys. Lett. 50, 1739 (1987).

${ }^{10}$ D. J. Chadi, Phys. Rev. Lett. 59, 1691 (1987); O. L. Alerhand, J. D. Joannopoulos, and D. Vanderbilt (unpublished).

${ }^{11}$ T. Sakamoto and G. Hashiguchi, Jpn. J. Appl. Phys. 25, L57 (1986).

${ }^{12}$ The competing model of D. E. Aspnes and J. Ihm [Phys. Rev. Lett. 57, 3054 (1986)] is found to be higher in energy by $0.3 \mathrm{eV}$ per unit length $(a \sqrt{2})$ along the step, and to be inconsistent with recent experimental evidence [P. E. Wierenga, J. A. Kubby, and J. E. Griffith, Phys. Rev. Lett. 59, 2169 (1987)].

${ }^{13}$ R. D. Bringans, R. I. G. Uhrberg, R. Z. Bachrach, and J. E. Northrup, Phys. Rev. Lett. 55, 533 (1985).

${ }^{14}$ R. I. G. Uhrberg, R. D. Bringans, R. Z. Bachrach, and J. E. Northrup, Phys. Rev. Lett. 56, 520 (1986).

${ }^{15} \mathrm{~L}$. Pauling, The Nature of the Chemical Bond (Cornell Univ. Press, Ithaca, NY, 1960), p. 93.

${ }^{16}$ D. J. Chadi, Phys. Rev. B 29, 785 (1984).

${ }^{17}$ W. A. Harrison, Electronic Structure and the Properties of Solids (Freeman, San Francisco, CA 1980).

${ }^{18}$ S. L. Wright, H. Kroemer, and M. Inada, J. Appl. Phys. 55, 2916 (1984); P. N. Uppal and H. Kroemer, ibid. 58, 2195 (1985). 